\title{
Interdependent Components of Generating Systems in the Theory of Pseudo-Holomorphic Functions
}

\author{
C. WITHALM
}

In many studies concerning the theory of pseudo-holomorphic functions in the sense of $L$. Bers the interdependence of the components of a relevant generating system turns out to be essential to investigate, e.g. inquiring into periodicity problems with respect to corresponding derivatives but also into multiple reasonable configurations.

KEY WORDS: pseudo-holomorphic functions, distinctive generating systems, singularities.

AMS(MOS)SUBJ.Class.: 30A93, 30G20, 35J45

\section{Preliminaries}

1.1. L. Bers [1-3] pointed out that the solutions of the homogeneous Vekua equation $w_{\bar{z}}-a w-b \bar{w}=0[9]$, by virtue of a fixed chosen generating system, say $E:=\langle F, G\rangle$, accomplishing $\operatorname{Im}(\bar{F} G)>0$ in some domain $D_{0} \subset \mathbb{C}$ and $F, G \in H_{D_{0}}^{1}$ (Hölder-continuously differentiable there) is uniquely representable by $w(z)=F(z) \varphi(z)+G(z) \psi(z)$ wherein $\varphi$ and $\psi$ are real-valued functions in $D \subset D_{0}$. Analogous to the classical theory we establish the differential operator $d_{E} / d z: w$ is called complex differentiable modulo $E$ at $z_{0} \in D$ if there $w(z)$ is complex linear approximable modulo $E$, i.e. if

$$
w(z)=F(z) \varphi\left(z_{0}\right)+G(z) \psi\left(z_{0}\right)+\lambda\left(z-z_{0}\right)+\rho\left|z-z_{0}\right|, \lim _{\substack{\left|z-z_{0}\right| \rightarrow 0 \\ z \neq \neq}} \rho\left(z, z_{0}\right)=0 .
$$

Hereby the complex $E$-derivative $\lambda=\dot{w}\left(z_{0}\right)=d_{E} w / d z$ of $w(z)$ at $z_{0}$ is defined, and obviously the complex linear approximability modulo $E$ of $w(z)$ is equivalent to the existence of $\lim _{z \rightarrow z_{0}} \frac{w(z)-F(z) \varphi\left(z_{0}\right)-G(z) \psi\left(z_{0}\right)}{z-z_{0}}=\lambda=\dot{w}\left(z_{0}\right)=\frac{d E w}{d z}\left(z_{0}\right){ }^{1)}$ If $d_{E} w / d z$ anywhere in $D$ does exist properly $w$ there is called a pseudo-holomorphic function modulo $E$ of the first kind or shortly an E-pseudo-holomorphic function.

The set of all $E$-pseudo-holomorphic functions defined in $D$ obviously forms an additive vector space upon $\mathbb{R}$, say $P_{D}(E)$, the subspace of all so-called generalized constants modulo $E$ with $\varphi, \psi \equiv$ const, shall be designed by $P_{D}^{0}(E)$ (cp. Remark $\left.4,(i i i)\right)$.

1.2. In generalization of the classical theory in some neighbourhood of $z_{0}, U=U\left(z_{0}\right) \subset D$, there hold the following statements:

1) More suggestive $\lim _{x \rightarrow x_{0}} \frac{F(x)\left(\varphi(x)-\varphi\left(x_{0}\right)\right)+G(x)\left(\psi^{2}(x)-\varphi\left(x_{0}\right)\right)}{x_{0}}$.

C. Withalm: Karl-Franzens-Universität, Institut für Mathematik, Heinrichstr. 36, A-8010 Graz ISSN $0232-2064 / \$ 2.50$ (C) Heldermann Verlag Berlin 
(i) If $w \in P_{U}(E)$, then $\varphi_{z}, \varphi_{\bar{z}}, \psi_{z}, \psi_{\bar{z}}$ exist at $z_{0}$ and

$$
F \varphi_{z}+G \psi_{z}=\dot{w}, \quad F \varphi_{\bar{z}}+G \psi_{\bar{z}}=0
$$

is valid there. (Generalized Cauchy-Riemann differential equations.)

(ii) If $\varphi, \psi \in C_{U}^{1}$ (continuously differentiable) and $F \varphi_{\bar{z}}+G \psi_{\bar{z}}=0$ is true there,then $w \in P_{U}(E)$ holds. (Generalization of the theorem of Loomann-Menchhoff.)

Remark 1: (i) Obviously $F, G \in P_{D}^{0}(E)$ and via

$$
F_{\bar{z}}=a F+b \bar{F}, G_{\bar{z}}=a G+b \bar{G}, F_{z}=A F+B \bar{F}, G_{z}=A G+B \bar{G}
$$

we obtain the so-called characteristic coefficients

$$
\begin{array}{ll}
a=: a_{E}=-\left(\bar{F} G_{\bar{z}}-F_{\bar{z}} \bar{G}\right) / \Delta_{E}, & b=: b_{E}=\left(F G_{\bar{z}}-F_{\bar{z}} G\right) / \Delta_{E} \\
A=: A_{E}=-\left(\bar{F} G_{z}-F_{z} \bar{G}\right) / \Delta_{E}, & B=: B_{E}=\left(F G_{z}-F_{z} G\right) / \Delta_{E}
\end{array}
$$

with $\Delta_{E}:=\operatorname{det}\left(\begin{array}{cc}F & G \\ \bar{F} & \bar{G}\end{array}\right)$. (The index " $E$ "is omittable if the meaning is clear!) Note that

$$
b_{E}=\left(\frac{G}{F}\right)_{z} \cdot \frac{F^{2}}{\Delta_{E}}, \quad B_{E}=\left(\frac{G}{F}\right)_{z} \cdot \frac{F^{2}}{\Delta_{E}}
$$

which gives a first hint at the importance of the interdependence of $F$ and $G$; too.

(ii) Note that conversely for any couple of bounded Hölder continuous functions $a, b$ defined in $D_{0}$ there exists a generating system $E$ such that $a=a_{E}$ and $b=b_{E}$.

Thus in $U=U\left(z_{0}\right) \subset D$ there hold the following statements:

(i) If $w \in P_{U}(E)$, then $w_{z}, w_{\bar{z}}$ exist at $z_{0}$ and

$$
w_{z}-A w-B \bar{w}=\dot{w}^{2)}, \quad w_{\bar{z}}-a w-b \bar{w}=0
$$

is valid there.

(ii) If $w \in C_{U}^{1}$ and $w_{\bar{z}}-a w-b \bar{w}=0$ is true there, then $w \in P_{U}(E)$ holds.

1.3. Before inquiring some F-G-interdependences a few basic results of the theory of pseudo-holomorphic functions shall be listed.

Remark 2: If $E=\langle F, G\rangle \equiv\langle 1, i\rangle=: I$, then the theory of pseudo-holomorphic functions obviously leads to the classical theory. Thus $P_{D}(I)$ designes the algebra (upon $\mathbb{K})$ of the holomorphic functions defined in $D$.

Remark 3: (i) $w \in P_{D}^{0}(E)$ is equivalent to $w(z) \equiv 0$ in $D$.

\footnotetext{
2) Pseudo-differential equation.
} 
(ii) If $w=F \varphi+G \psi \in P_{D}(E)$, then the so-called pseudo-holomorphic function modulo $E$ of the second kind $\varphi+i \psi$ is a $H_{D}^{2}$-function (being a $K$-quasiconformal mapping). Let $w$ be any function of the structure $F \varphi+G \psi$ with $E=\langle F, G\rangle$ being a generating system and $\varphi, \psi$ arbitrary ( $w$ not necessarily an element of $P_{D}(E)$ ). Then the 1-1-operator $p_{E}$ shall be defined by virtue of $p_{E} w \equiv p_{E}(F \varphi+G \psi)=\varphi+i \psi$.

(iii) If $w \in P_{D}(E)$, then $\dot{w}$ is a $H_{D}^{1}$-function and $\dot{w}_{\bar{z}}=a_{E} \dot{w}-B_{E} \overline{\bar{w}}$ holds in $D$. Thus $\dot{w}$ is a pseudo-holomorphic function modulo a generating system $E^{1}:=\left\langle F_{1}, G_{1}\right\rangle$, called the successor of $E=: E^{0}\left(E^{0}\right.$ predecessor of $\left.E^{1}\right)$ and defined by virtue of $a_{E^{1}}=a_{E^{0}}$, $b_{E^{\mathfrak{l}}}=-B_{E^{\circ}}$. Iteratively we obtain a corresponding generating sequence $\left(E^{\nu}\right)_{\nu \in Z}$ which $E^{0}$ is embedded in such that (involving derivatives of all orders) $d_{E^{\nu}} / d z$ maps $P_{D}\left(E^{\nu}\right)$ into $P_{D}\left(E^{\nu+1}\right)$. If for a fixed $\mu \in \mathbb{N}$ always $E^{\nu+\mu}=E^{\nu}, \mu$ is called the period of $\left(E^{\nu}\right)_{\nu \in Z}$. (The relating periodicity problem was solved by M.H. Protter [6].)

\section{F-G-interdependences}

2.1. In the well-improved theory of pseudo-holomorphic functions which was developed by L. Bers and his school (similar to Vekua's investigations on generalized analytic functions, using some different approach) the mutual correlation of the components of the generating system $E=\langle F, G\rangle$ plays an important role in considering many fundamental examinations (e.g. Remark 1,(i)). Some corresponding aspects shall be presented here.

A prominent theorem was already proved by L. Bers in the beginning fifties.

Theorem 1 (L. Bers): Let $w=F \varphi+G \psi \in P_{D}(E)$. Then the minimal period $\mu$ of the generating sequence which $E=E^{0}$ is embedded in is equal to 1 if and only if the ratio $G / F$ depends on $y$ only.

The proof of that theorem according to the definition in Remark 3,(iii) is based on $b_{E^{1}}=-B_{E^{0}}$ which leads to the relation $(G / F)_{\bar{z}}+(G / F)_{z}=0$ (cp. Remark 1,(i)).

2.2. For considering a few additional reasonable properties of dependence of $F$ and $G$ we set $w=F \varphi+G \psi=F\left(\varphi+\frac{G}{F} \psi\right)=: F w_{N}$ (where the index $N$ vaguely shall remind the reader of "norm" in the sense of standardize). Thus similar to the definition of the" $p_{E}$-operator (Remark 3,(ii)) we shall define the 1-1-operator $N_{E}$ by virtue of $N_{E} w=$ $N_{E}(F \varphi+G \psi)=\varphi+\frac{G}{F} \psi=w_{N_{E}}$ wherein again $E=\langle F, G\rangle$ is a generating system but not necessarily $w \in P_{D}(E)$. (Using " $N_{E}$ " as an index we shall write a pure " $N$ " if not any confusion is possible.)

Comparing Subsection 1.1 it can be shown that $w_{N} \in P_{D}\left(\left\langle 1, \frac{G}{F}\right\rangle\right)$ : for if $E=\langle F, G\rangle$ is a generating system in $D_{0} \supset D$, then there $\left(1, \frac{G}{F}\right)=: E^{N}$ is a generating system, too, because of the facts that $0<\operatorname{Im}(\bar{F} G)=\operatorname{Im}\left(\bar{F} \frac{G}{F} F\right)=|F|^{2} \operatorname{Im}\left(\overline{1} \frac{G}{F}\right)$ and that $F, G \in H_{D_{0}}^{1}$ implies $\frac{G}{F} \in H_{D_{0}}^{1}$; from $F \varphi_{\bar{z}}+G \psi_{\bar{z}}=0$ we obtain $\varphi_{\bar{z}}+\frac{G}{F} \psi_{\bar{z}}=0$ which proves our assertion (cp. (1)). Note that $p_{E} w=p_{E^{N}} w_{N}$. 
.In this paper we shall discuss the cases $G / F \in P_{D_{0}}(I)$ (cp. Remark 2) and $G / F \in$ $P_{D_{0}}(\bar{I}), P_{D_{0}}(\bar{I})$ being the set of all anti-holomorphic functions defined in the domain $D_{0}$.

Theorem 2: Let $w=F \varphi+G \psi \in P_{D}(E)$ and $G / F \in P_{D_{0}}(I)$ with $D \subset D_{0}$. Then $w_{N} \in P_{D}(I)$.

Proof: From $w \in P_{D}(E)$ it follows $w_{N} \in P_{D}\left(E^{N}\right)$ such that $\varphi_{\bar{z}}+\frac{G}{F} \psi_{\bar{z}}=0$. Because of $G / F$ being holomorphic we obtain $\left(\varphi+\frac{G}{F} \psi\right)_{\bar{z}}=0$.

Thus by definition of $N_{E}$ under the assumptions of Theroem 2 we can write $N_{E}$ : $P_{D}(E) \rightarrow P_{D}(I)$.

Remark 4: (i) $w_{N}=\varphi+\frac{G}{F} \psi$ being holomorphic, $w=F w_{N}$ already is one possible appearance of $w$ according to the similarity principle (L. Bers) or the formula of reciprocity (I.iN. Vekua [9]), which says that $w=F \varphi+G \psi \in P_{D}(E)$ allows a representation of the structure $e^{s} f$ with $f$ being holomorphic in $D$ and (defining the $T_{D}$ integral operator by virtue of

$$
\left(T_{D} h\right)(z)=-\frac{1}{\pi} \iint_{D} \frac{h(\zeta)}{\zeta-z} d \xi d \eta
$$

as usual) $s(z)=T_{D}(a+b \bar{w} / w)$ et vice versa. Thus in the upper case $F$ plays the part of a suitable exponential function $e^{s}$. Setting $w_{N}=u+i v$ we obtain $F u_{\bar{z}}+i F v_{\bar{z}}=0$ such that $w=F w_{N} \in P_{D}(\langle F, i F\rangle)(\langle F, i F\rangle$ obviously is a generating system).

(ii) In general $E$ and $\tilde{E}$ are called equipotent generating systems relative to $W$ if $P_{D}(E) \ni W \in P_{D}(\tilde{E})$ such that in the objective case $\langle F, G\rangle$ and $\langle F, i F\rangle$ are equipotent generating systems relative to $w$, or more general: if $w=F \varphi+G \psi=e^{s} f$, then $\langle F, G\rangle$ and $\left\langle e^{s}, i e^{s}\right\rangle$ are equipotent generating systems relative to $w$. Because of the elementary consequences generating systems of the structure $\left\langle e^{s}, i e^{s}\right\rangle$ (or $\langle F, i F\rangle$, respectively) are called main generating systems.

(iii) If in $w=e^{s} f$ the function $f$ is a pure constant, then L. Bers calls $w$ a generalized constant; but because of $w$ generally is interpretable as an element of $P_{D}\left(\left\langle e^{s}, i e^{s}\right\rangle\right)$ we use the relevant definition given in Subsection 1.1 (cp., e.g., [10] and Remark 3,(i)).

Remark 5: Finally note that $(G / F)_{\bar{z}}=0$ implies $b_{E}=0$. (cp. Remark 1).

2.3. Let $f=u+i v$ be an element of the algebra $P_{D_{0}}(\bar{I})$, i.e. $f_{z}=0$ or $u_{x}=-v_{y}, u_{y}=v_{x}$, respectively.

'Theorem 3: Let $E=\langle F, G\rangle$ be a generating system in $D_{0}$ and let $G / F \in P_{D_{0}}(\bar{I})$. Then for each $w=F \varphi+G \psi \in P_{D}(E), D \subset D_{0}$, there exists a function $\tilde{w}=F \tilde{\varphi}+\dot{G} \tilde{\psi} \in$ $P_{D}(E)$ such that $\Phi:=\varphi+i \tilde{\varphi}$ and $\Psi:=\psi+i \tilde{\psi}$ are holomorphic in $D$ (see [12]). 
Proof: Defining. $-i G / F=: \sigma+i \tau$ and $-i F / G=: \hat{\sigma}+i \hat{\tau}$ the relation $F \dot{\varphi}_{\bar{z}}+G \psi_{\bar{z}}=0$ is equivalent to the system

$$
\begin{array}{ll}
\varphi_{x}=\tau \psi_{x}+\sigma \psi_{y}, & \varphi_{y}=-\sigma \psi_{x}+\tau \psi_{y} \\
\psi_{x}=\hat{\tau} \varphi_{x}+\hat{\sigma} \varphi_{y}, & \psi_{y}=-\hat{\sigma} \varphi_{x}+\hat{\tau} \varphi_{y}
\end{array}
$$

from which by a repeated differentiation (being legitimate, cp. Remark 3,(ii)) and by summing up we gain the system

$$
\Delta \varphi+\hat{\alpha} \varphi_{x}+\hat{\beta} \varphi_{y}=0, \quad \Delta \psi+\alpha \psi_{x}+\beta \psi_{y} \stackrel{\therefore}{=} 0
$$

wherein

$$
\alpha=\frac{\sigma_{x}+\tau_{y}}{\sigma}, \quad \hat{\alpha}=\frac{\hat{\sigma}_{x}+\hat{\tau}_{y}}{\hat{\sigma}} \text { and } \beta=\frac{\sigma_{y}-\tau_{x}}{\sigma}, \hat{\beta}=\frac{\hat{\sigma}_{y}-\hat{\tau}_{x}}{\hat{\sigma}}
$$

which means that $\alpha, \beta, \hat{\alpha}, \hat{\beta}$ vanish if $-i G / F$ (and so $-i F / G$ ) are anti-holomorphic. As in the classical theory one finds the supplements $\tilde{\varphi}$ and $\tilde{\psi}$ (conjugated harmonic to $\varphi$ and $\psi$, respectively) at which $F \tilde{\varphi}+G \tilde{\psi}$ turns out to be an element of $P_{D}(E)$ again. (Note that $i F \tilde{\varphi}+i G \tilde{\psi} \in P_{D}(i E)$.)

Remark 6: (i) If conversely $\varphi$ and consequently $\psi$ (et vice versa) satisfy a (nontriviall) Laplace equation, from $w_{N}=\varphi+\frac{G}{F} \psi \in P_{D}\left(E^{N}\right)$ and thus $\varphi_{\bar{z}}+\frac{G}{F} \psi_{\bar{z}}=0$ we obtain $\varphi_{\bar{z} z}+\left(\frac{G}{F}\right)_{z} \psi_{\bar{z}}+\left(\frac{G}{F}\right) \psi_{\bar{z} z}=0$, hence $\left(\frac{G}{F}\right)_{z}=0$. Consequently by a suitable formulation Theorem 3 is necessary and sufficient.

(ii) Note that every complex-valued potential function being regular in some domain $\left\{z: \operatorname{Re}\left(z-z_{0}\right)<a, \operatorname{Im}\left(z-z_{0}\right)<b\right\}$ can be written as the sum of a holomorphic and an anti-holomorphic function.

Theorem 4: Under the premises of Theorem $3 N_{E^{1}} \frac{d \varepsilon}{d z}: P_{D}(E) \rightarrow P_{D}(I)$ is true for $E^{1}$ being a successor of $E=E^{0}$ in $D_{0} \subset D$.

Proof: From $(G / F)_{z}=0$ we obtain $B_{E}=0$ (cp. Remark 1,(i)). According to the definition in Remark 3,(iii) from $b_{E^{1}}=-B_{E^{0}}$ it follows $b_{E^{1}}=0$ which implies $G_{1} / F_{1} \in$ $P_{D_{0}}(I)$. Thus the proof is accomplished by Remark 3,(ii) again and by, Theorem 2.

Remark 7: Note that $w=F \varphi+G \psi \in P_{D}(E)$ implies $w_{1}:=\dot{w}=F_{1} \varphi_{1}+G_{1} \psi_{1} \in$ $P_{D}\left(E^{1}\right)$ but $d_{E^{N}} w_{N} / d z$ is not equal to $w_{1 N}$ in general.

\section{Integral operators}

3.1. With regard to the underlying Vekua equation $w_{\bar{z}}-a w-b \bar{w}=0$ or the pseudodifferential equation $w_{z}-A w-B \bar{w}=\dot{w}$, respectively (cp. (2)) it is useful to establish some relevant features concerning $w_{N}$. 
First it is easy to compute (cp. Remark 1,(i)) that

$$
a_{E^{N}}=-b_{E} \frac{\bar{F}}{F}, \quad b_{E^{N}}=b_{E} \frac{\bar{F}}{F}, \quad A_{E^{N}}=-B_{E} \frac{\bar{F}}{F}, \quad B_{E^{N}}=B_{E} \frac{\bar{F}}{F}
$$

are the characteristic coefficients for the generating system $E^{N}$ such that the correlative Vekua equation and the pseudo-differential equation, respectively, reduce to

$$
\left(w_{N}\right)_{\bar{z}}+b_{E} \frac{\bar{F}}{F} \cdot 2 i \operatorname{Im} \cdot w_{N}=0,\left(w_{N}\right)_{z}+B_{E} \frac{\bar{F}}{F} \cdot 2 i \operatorname{Im} w_{N}=\dot{w}_{N}
$$

If specifically $G / F \in P_{D_{0}}(I)$, because of $b_{E}=0$ it follows of course $w_{N}$ to be holomorphic (cp. Theorem 2 and Remark 5). If specifically $G / F \in P_{D_{0}}(\bar{I})$ because of $B_{E}=0$ it follows $\left(w_{N}\right)_{z}=\dot{w}_{N}=d_{E^{N}} w_{N} / d z$. (Note that $\dot{w}_{N}=\varphi_{z}+\frac{G}{F} \psi_{z}=\left(\varphi+\frac{G}{F} \psi\right)_{z}=\left(w_{N}\right)_{z}$, cp. (1).)

3.2. Considering boundary value problems, pseudo-holomorphic functions modulo a generating system of the structure $\langle 1, H\rangle$ play a remarkable part; that follows from the configuration of the differential equations (3) (canonical form) being a basis to investigate correlative problems. Note that the definition of the corresponding coefficients $\alpha, \beta, \hat{\alpha}, \hat{\beta}$ depends on the ratio $G / F$ again, and that for $\langle 1, G / F\rangle$ by definition of $\sigma, \tau, \hat{\sigma}, \hat{\tau}$ in the proof of Theorem 3 we can use the same abbreviations - obtaining an equivalent system to (3). See [1].

3.3. Let $E=\langle F, G\rangle$ be a generating system defined in $D_{0} \subset \mathbb{C}$, let $\Gamma=\Gamma\left[z_{0}, z\right] \subset D \subset D_{0}$ be a path of integration, and let $W$ be a function continuous on $\Gamma$. Then

$$
p_{E} \int_{\Gamma\left[z_{0}, z\right]} W d_{E} \zeta:=\operatorname{Re} \int_{\Gamma\left[z_{0}, z\right]} G^{*} W d \zeta+i \operatorname{Re} \int_{\Gamma\left[z_{0}, z\right]} F^{*} W d \zeta
$$

is called the $p_{E}$-integral of $W$ over $\Gamma$ wherein $F^{*}:=-2 \bar{F} / \Delta_{E}$ and $G^{*}:=2 \bar{G} / \Delta_{E}$ and $E^{*}=\left\langle F^{*}, G^{*}\right\rangle$ is the generating system adjoint to $E$. (Note that indeed $E^{*}$ is a generating system anew:) Moreover

$$
\int_{\Gamma\left[x_{0}, z\right]} W d_{E} \zeta:=F(z) \operatorname{Re} \dot{p}_{E} \int_{\Gamma\left[z_{0}, z\right]} W d_{E} \zeta+G(z) \operatorname{Im} \dot{p}_{E} \int_{\Gamma\left[z_{0}, z\right]}^{\dot{W}} \dot{W} d_{E} \zeta
$$

is called the $E$-integral of $W$ over $\Gamma$.

Remark 8: Note that in contrast to the $p_{E}$-integral the $E$-integral is no additive functional of the path of integration.

Remark 9: (i) If specifically $\dot{w}=F \varphi+G \psi \in P_{D}(E)$, then $\dot{w}=d_{E} w / d z$ is $p_{E^{-}}$and $E$-integrable as well and

$$
\begin{aligned}
p_{E} \int_{\Gamma\left[z_{0}, z\right]} \dot{w} d_{E} \zeta & =\left(\varphi(z)-\varphi\left(z_{0}\right)\right)+i\left(\psi(z)-\psi\left(z_{0}\right)\right)=p_{E}\left(w(z)-w\left(z_{0}\right)\right) \\
\int_{\Gamma\left[z_{0}, z\right]} \dot{w} d_{E} \zeta & =F(z)\left(\varphi(z)-\varphi\left(z_{0}\right)\right)+G(z)\left(\psi(z)-\psi\left(z_{0}\right)\right) \\
& =w(z)-\left(F(z) \varphi\left(z_{0}\right)+G(z) \psi\left(z_{0}\right)\right)
\end{aligned}
$$


(ii). Setting $W=F W_{N}, W_{N}$ being some suitable function and $F$ naturally being the first component of $E$, we obtain the formulas

$$
p_{E^{N}} \int_{\Gamma\left[z_{0}, z\right]} W_{N} d_{E^{N}} \zeta=p_{E} \int_{\Gamma\left[z_{0}, z\right]} W d_{E} \zeta, \quad F(z) \int_{\Gamma\left[z_{0}, z\right]} W_{N} d_{E^{N}} \zeta=\int_{\Gamma\left[z_{0}, z\right]} W d_{E} \zeta
$$

which result from the fact that $\left(E^{N}\right)^{*}=\left\langle 1^{*},(G / F)^{*}\right\rangle=\left\langle F F^{*}, F G^{*}\right\rangle$. Formulas correlativ to (i) obviously we obtain for $W_{N}=\dot{w}_{N}=d_{E^{N}} w_{N} / d z$ (cp. Remark 7).

Theorem 5 (L. Bers): Let $E^{1}$ be a successor of $E^{0}=E$. Then every $E^{1}$-pseudoholomorphic function is an $E$-derivative (of a not necessarily single-valued function).

Thus the statements of the present Subsection 3.3 should be formulated with regard to Theorem 5. Before considering some consequences we are to investigate several properties of the derivatives of anti-holomorphic depending components of a generating system.

\section{Derivating E :}

4.1. Generally let $w=F \varphi+G \psi \in P_{D}(E)$. Then from $0=F \varphi_{\bar{z}}+G \psi_{\bar{z}}$ (respectively $0=\bar{F} \varphi_{z}+\bar{G} \psi_{z} ; \varphi, \psi$ real $)$ and $\dot{w}=F \varphi_{z}+G \psi_{z}$ it follows

$$
\varphi_{z}=\frac{\bar{G} \dot{w}}{\Delta_{E}}=\frac{G^{*} \dot{w}}{2}, \quad \psi_{z}=-\frac{\bar{F} \dot{w}}{\Delta_{E}}=\frac{F^{*} \dot{w}}{2}
$$

Remember that $\varphi, \psi \in H_{D}^{2}$, see Remark 3,(ii). Now let $G / F \in P_{D_{0}}(\bar{I})$ which implies that $\alpha, \beta, \hat{\alpha}, \hat{\beta}$ vanish in (3) such that $\Delta \varphi \doteq 0=\Delta \psi$; hence, because of

$$
\left(\frac{\bar{G} \dot{w}}{\Delta_{E}}\right)_{\bar{z}}=\left(\frac{G^{*} \dot{w}}{2}\right)_{\bar{z}}=0, \quad\left(-\frac{\bar{F} \dot{w}}{\Delta_{E}}\right)_{\bar{z}}=\left(\frac{F^{*} \dot{w}}{2}\right)_{\bar{z}}=0
$$

we learn the holomorphy of $G^{*} \dot{w}$ and $F^{*} \dot{w}$ in $D \subset D_{0}$.

4.2. Let again $w=F \varphi+G \psi \in P_{D}(E)$ and $G / F \in P_{D_{0}}(\bar{I}), D \subset D_{0}$. First of all from $E \in H_{D_{0}}^{1} \times H_{D_{0}}^{1}{ }^{\prime}\left(\mathrm{cp}\right.$. Subsection 1.1) and $0=F \varphi_{\bar{z}}+G \psi_{\bar{z}}$, by (4) and (5), respectively, ensues $0=F_{z} \varphi_{z}+G_{z} \psi_{z}$ such that probably $W_{1}:=F_{z} \varphi+G_{z} \psi \in P_{D}\left(E_{z}\right)$. Indeed presuming $E \in H_{D_{0}}^{2} \times H_{D_{0}}^{2}$ on account of $F_{z}=A F+B \bar{F}, G_{z}=A G+B \bar{G}$ (cp. Remark 1,(i)) and $B \equiv 0$ (see Theorem 4) we obtain $\operatorname{Im}\left(\bar{F}_{z} G_{z}\right)=\operatorname{Im}(\bar{A} \bar{F} A G)=|A|^{2} \operatorname{Im}(\bar{F} G)>0$, (respecting eventual zeros of $A$ in $D_{0}$ ), hence $W_{1}=A w \in P_{D}(A E) \equiv P_{D}\left(E_{z}\right)$. Note that $N_{E} w=N_{E}(F \varphi+G \psi)=\varphi+\frac{G}{F} \psi \in P_{D}\left(E^{N}\right)$ and $N_{E_{z}} W_{1} \doteq N_{E_{z}}\left(F_{z} \varphi+G_{z} \psi\right)^{\prime}=$ $N_{A E}(A F \varphi+A G \psi)=\varphi+\frac{G}{F} \psi \in P_{D}\left(E^{N}\right)$ as well.

Presuming $E \in H_{D_{0}}^{3} \times H_{D_{0}}^{3}$ and considering that $0=F_{z} \varphi_{\bar{z}}+G_{z} \psi_{\bar{z}}$ implies $0=F_{z z} \varphi_{\bar{z}}+$ $G_{z z} \psi_{\bar{z}}$ we analogously obtain $F_{z z}=A_{z} F+A F_{z}=\left(A_{z}+A^{2}\right) F$ and $G_{z z}=\left(A_{z}+A^{2}\right) G$, 
respectively, such that from $\operatorname{Im}\left(\overline{F_{z z}} G_{z z}\right)=\left|A_{z}+A^{2}\right|^{2} \operatorname{Im}(F G)>0$ (again regarding possible zeros of the factors at $E$ in $\left.D_{0}\right)$ we get $W_{2}:=F_{z z} \varphi+G_{z z} \psi$ as an element of $P_{D}\left(\left(A_{z}+A^{2}\right) E\right)$ or of $P_{D}\left(E_{z z}\right)$, respectively, where again $N_{E_{z}} W_{2}=\varphi+\frac{G}{F} \psi \in P_{D}\left(E^{N}\right)$, and so on.

Thus we have proved

" Theorem 6: Let $E \in H_{D_{0}}^{\nu+2} \times H_{D_{0}}^{\nu+2}, \nu=0,1,2, \ldots, w=F \varphi+G \psi \in P_{D}(E)$ and $G / F \in P_{D_{0}}(\bar{I})$. Then $d^{\nu} E / d z^{\nu}$ is a generating system with $\frac{d^{\nu} G}{d z^{\nu}} / \frac{d^{\nu} F}{d z^{\nu}}=G / F, W_{\nu}=$ $\frac{d^{\nu} F}{d z^{\nu}} \varphi+\frac{d^{\nu} G}{d z^{\nu}} \psi \in P_{D}\left(d^{\nu} E / d z^{\nu}\right)$ and $N_{d^{\nu} E / d z^{\nu}} W_{\nu}=N_{E} w=w_{N} \in P_{D}\left(E^{N}\right)$, omitting eventual zeros of the factors at $E$ in $D_{0}$.

Note that all $W_{\nu}$ involve the same pseudo-holomorphic functions of the second kind!

Remark 10: If $E$ is a generating system for a suitable $H$ (see the conditions in Subsection 1.1), also $H E=: \tilde{E}$ is a generating system too. In that case $E$ and $\tilde{E}$ are called similar generating systems (L. Bers). Of course by hypotheses of Theorem $6 d^{\nu} E / d z^{\nu}$ for all $\nu$ are similar generating systems in pairs. Note that $w \in P_{D}(E)$ and $\tilde{w}=H w \in P_{D}(\tilde{E})$ imply $p_{E} w=p_{\tilde{E}} \tilde{w}$ and $N_{E} w=N_{\tilde{E}} \tilde{w}$.

Let be $E^{*}=\left\langle F^{*}, G^{*}\right\rangle=\left\langle-\frac{2 \tilde{F}}{\Delta_{E}}, \frac{2 \bar{G}}{\Delta_{E}}\right\rangle$ the adjoint generating system to $E=\langle F, G\rangle$ (cp. Subsection 3.3). Then it is easy to check that for $G / F \in P_{D_{0}}(\bar{I})$ we obtain $F_{\bar{z}}^{*}=-a_{E} F^{*}-$ $\bar{B}_{E} \bar{F}^{*}=-a_{E} F^{*}$ and $G_{\bar{z}}^{*}=-a_{E} G^{*}$ such that for $E \in H_{D_{0}}^{2} \times H_{D_{0}}^{2}-$ like above $-\left\langle F_{\bar{z}}^{*}, G_{\bar{z}}^{*}\right\rangle=$ $E_{\bar{z}}^{*}$ turns out to be a generating system, and so on. Thus for corresponding $E^{*}$-pseudoholomorphic functions a statement similar to Theorem 6 obviously can be formulated.

Note that $G / F \in P_{D}(\bar{I})$ implies $G^{*} / F^{*}=-\bar{G} / \bar{F} \in P_{D}(I)$ such that for those $E^{*}$ pseudo-holomorphic functions Theorem 2 becomes valid.

\section{Cauchy's theorems}

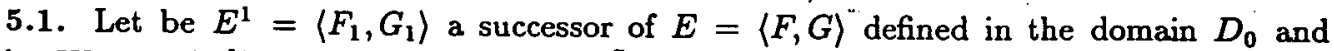
let $W \in P_{D}\left(E^{1}\right)$ (cp. Theorem 5) with $\tilde{D} \subset \subset D \subset D_{0}$ wherein $\tilde{D}$ is a simply connected domain and $\partial \tilde{D}$ being a path of integration. Then L. Bers proved $\int_{\partial \tilde{D}} W d_{E} \zeta=0$ applying Green's identity

$$
\int_{\partial \bar{D}} F^{*} W d \zeta=2 i \iint_{\bar{D}}\left(F^{*} W\right)_{\bar{\zeta}} d \xi d \eta
$$

and showing that the integral on the right-hand side is a pure real number. Thus by definition (cp. Subsection 3.3) the proof is finished by deliberating that the same argument holds for $\int_{\partial \bar{D}} G^{*} W d \zeta$. But note that by that definition we obtain $\int_{\partial \bar{D}} W d_{E} \zeta=F(z) \cdot O+$ $G(z) \cdot O$ wherein $z$ may by any point on the boundary of $\tilde{D}$. Thus interpreting $\int_{\partial \tilde{D}(z)} W d_{E} \zeta$ as a function in respect of the (upper bound) variable $z \in \partial \tilde{D}$ (mind Remark 8) we more 
exactly should expound that integral as the neutral element of $P_{D}^{0}(E)$ restricted onto $\partial \tilde{D}$. Consequently regarding a family of simply connected subdomains $\bar{D}_{\kappa}, 0<\kappa \leq 1$, being an exhausting of $\tilde{D}$, i.e. $\tilde{D}_{1}=\tilde{D}$ and $\tilde{D}_{\kappa}^{\prime} \subset \subset \bar{D}_{\kappa}$ if $\kappa^{\prime}<\kappa$, where $\operatorname{dist}\left(\tilde{D}_{\kappa^{\prime}}, \partial \tilde{D}_{\kappa}\right) \geq$ const $/\left(\kappa-\kappa^{\prime}\right)$, and respecting the consideration above, $z$ varying on $\partial \tilde{D}_{\kappa}$, naturally the neutral element of $P_{\bar{D}}^{0}(E)$ is generated (cp. Subsection 1.1).

5.2. Now let $w=F \varphi+G \psi \in P_{D}(E)$ and let $G / F \in P_{D_{0}}(\bar{I})$ again. From (5) we know that $G^{*} \dot{w}$ and $F^{*} \dot{w}$ turn out to be holomorphic in the domain of definition such that by Cauchy's theorem $\int_{\partial \bar{D}} G^{*} \dot{w} d \zeta$ and $\int_{\partial \bar{D}} F^{*} \dot{w} d \zeta$ vanish (defining $\tilde{D}$ as above) without using any values of the integrands in $\tilde{D}$ of course (cp. Subsection 5.1). Naturally - taking account of the definitions in Subsection 3.3 - together with the $p_{E}$-integral the $E$-integral vanishes as well wherein $\int_{\partial \bar{D}(z)} \dot{w} d_{E} \zeta$ is interpretable as above.

5.3. Indeed, that interpretation is motivated considering the integral definitions in Subsection 3.3 being relevant for $W(\zeta):=\dot{w}(\zeta) /\left(\zeta-z_{0}\right)$ wherein $z_{0}$ denotes a fixed point of $\tilde{D}$ and $\zeta$ is the variable of integration. Naturally from $\dot{w}=F \varphi_{z}+G \psi_{z}$ and $0=F \varphi_{\bar{z}}+G \psi_{\bar{z}}$ we obtain $i \dot{w}=F \varphi_{y}+G \psi_{y}$. Applying Cauchy's integral formula with respect to Subsection 4.1 we get

$$
\operatorname{Re} \int_{\partial \tilde{D}} G^{*} \frac{\dot{w}}{\zeta-z_{0}} d \zeta=\operatorname{Re}\left[2 \pi i \cdot 2 \cdot \frac{1}{2}\left(\varphi_{x}-i \varphi_{y}\right)\left(z_{0}\right)\right]=2 \pi \varphi_{y}\left(z_{0}\right)
$$

and analogously

$$
i \operatorname{Re} \int_{\partial \bar{D}} F^{*} \frac{\dot{w}}{\zeta-z_{0}} d \zeta=i \operatorname{Re}\left[2 \pi i \cdot 2 \cdot \frac{1}{2}\left(\psi_{x}-i \psi_{y}\right)\left(z_{0}\right)\right]=2 \pi i \psi_{y}\left(z_{0}\right)
$$

which means that

$$
\frac{1}{2 \pi} p_{E} \int_{\partial \bar{D}} \frac{\dot{w}}{\zeta-z_{0}} d_{E} \zeta=\left(\varphi_{y}+i \psi_{y}\right)\left(z_{0}\right)=p_{E} i \dot{w}\left(z_{0}\right)^{3)}
$$

and

$$
\left.\frac{1}{2 \pi} \int_{\partial \dot{D}} \frac{\dot{w}}{\zeta-z_{0}} d_{E} \zeta=F(z) \varphi_{y}\left(z_{0}\right)+G(z) \psi_{y}\left(z_{0}\right)^{4}\right)
$$

wherein again $z$ may be any point on the boundary of $\tilde{D}$. Thus $\int_{\partial \tilde{D}(z)} \frac{\dot{w}}{\zeta-x_{0}} d_{E} \zeta$ more precisely chàracterizes a generalized constant modulo $E$ in $D \backslash\left\{z_{0}\right\}$ restricted onto $\partial \tilde{D}$, and an exhausting of $\tilde{D}$ correlative to Subsection 5.1 leads to an element of $P_{\tilde{D} \backslash\left\{x_{0}\right\}}^{0}(E)$; by continuation into $z_{0}$ we obtain an element of $P_{\tilde{D}}^{0}(E)$.

3) See the definition of the $p_{E}$-operator in Remark 3,(ii).

4) Note that for $\theta \dot{D} \rightarrow\left\langle z_{0}\right\rangle$ that expression tends to $i \dot{w}\left(z_{0}\right)$. 
5.4. In general let $z_{0} \in \tilde{D}$ contain any singularity of $\dot{w}$ the support of which being $p_{E} \dot{w}$. (Note that $w$ and simultaneously $\dot{w}$ have in some $z$ singularities of the same characteristic (L. Bers).) Then considerations near to those of Subsections $5.1-5.3$ show that

$$
\int_{\partial \tilde{D}(z)} \dot{w} d_{E} \zeta=F(z) \operatorname{Re}\left[2 \pi i \operatorname{res}_{z_{0}} G^{*} \dot{w}\right]+G(\dot{z}) \operatorname{Re}\left[2 \pi i \operatorname{res}_{z_{0}} F^{*} \dot{w}\right]
$$

generates an element at least of $P_{\bar{D} \backslash\left\{z_{0}\right\}}^{0}(E)$ anew.

\section{Remark}

Apparently F-G-interdependences in the theory of pseudo-holomorphic functions are not unessential to study. In the present paper only the simple case $G / F \in P_{D}(I)$ and the case $G / F \in P_{D}(\bar{I})$ was inquired for several function-theoretical configurations. It is a reasonable assumption that investigations on further correlative dependences (cp. Remark 6,(ii)) could mature interesting results.

\section{REFERENCES}

[1] Bers, L., Theory of pseudo-analytic functions, New York University, 1953.

[2] Bers, L., Local theory of pseudo-analytic functions, Lectures on Functions of a Complex Variable, University of Michigan Press, 1955, pp. 213-244.

[3] Bers, L., An outline of the theory of pseudo-analytic functions, Bull. Amer. Math. Sci. 62 No. 1 (1956), 291-331.

[4] Goldschmidt, B., Funktionentheoretische Eigenschaften der Lösungen der Vekuaschen Differentialgleichungen mit Koeffizienten in $L_{p, \text { loc }}(G)$, Math. Nachr. 84. (1978), 229- 246.

[5] Lanckau, E-and Tutschke, W., Eds., Complex Anatysis - Methods, Trends and Applications, Akademie-Verlag, Berlin, 1983.

[6] Protter, M.H., The periodicity problem for pseudo-analytic functions, Ann. of Math. (2) 64 (1956), 154-174.

[7] Tutschke, W., Partielle kömplexe Differentialgleichungen in einer und mehreren komplexen Variablen, Akademie-Verlag, Berlin, 1977.

[8] Tutschke, W. and Withalm, Cl., The Cauchy-Kovalevska theorem for pseudo-holomorphic functions in the sense of $L$. Bers, Complex Variables: Theory and Applications 1 (1983), 389-393.

[9] Vekua, I.N., Verallgemeinerte analytische Funktionen, Akademie-Verlag, Berlin, 1963.

[10] Withalm Cl., Über einen Integraloperator für pseudo-holomorphe Funktionen modulo eines Haupterzeugendensystems, Complex Variables: Theory and Applications 4 No. 2 (1985), 155-161. [11] Withalm Cl., Ed., Complex methods on partial differential equations - aspects in complex analysis,
Berlin, 1989.

[12] Withalm Cl. Supplierende pseudo-holomorphe Funktionen, Rostocker Mathematisches Kolloquium 40 $(1990), 19-28$. 